\title{
Necrose Isquêmica Hepática e Diabete Melito. Relato de Caso
}

\author{
Liver Ischemic Necrosis and Diabetes Mellitus. Case Report.
}

\author{
Ticiana Paes ${ }^{1}$, Fernanda Martins Gazoni', Nathanael de Freitas Pinheiro Junior², Hélio Penna Guimarães ${ }^{3,4,5}$, \\ Renato Delascio Lopes ${ }^{3}$, Valéria Pereira Lanzoni6, Letícia Sandre Vendrame ${ }^{3,5}$, Antônio Carlos Lopes ${ }^{7}$
}

\section{RESUMO}

JUSTIFICATIVA E OBJETIVOS: O infarto hepático é definido como necrose isquêmica do parênquima hepático envolvendo pelo menos dois ácinos. Trata-se de evento considerado raro devido ao duplo suprimento sangüíneo, arterial e venoso. O objetivo deste estudo foi relatar um caso de paciente não sabidamente diabética que desenvolveu extensas áreas isquêmicas de infarto hepático, após quadro de descompensação aguda da diabete.

RELATO DO CASO: Paciente do sexo feminino, 67 anos, hipertensa, procurou o Pronto Socorro com queixas de polidipsia, poliúria, turvação visual, náuseas e vômitos, dificuldade para deambular, havia aproximadamente 10 dias. Ao exame físico foi observado desidratação, palidez cutânea, cianose periférica, hipotermia, taquicardia, hipotensão, dor abdominal leve e difusa. Exames laboratoriais mostraram: leucócitos: 16800, creatinina $(\mathrm{Cr})$ : 3,7, uréia

\footnotetext{
1. Médica Especializanda da Disciplina de Clínica Médica da UNIFESP/EPM.

2. Médico Residente da Disciplina de Patologia Clínica da UNIFESP/EPM.

3. Médico Assistente da Disciplina de Clínica Médica da UNIFESP/ EPM e Coordenador do Núcleo de Estudos em Emergências Clínicas NEEC - Disciplina de Clínica Médica - UNIFESP/EPM.

4. Médico da Divisão de Pesquisa do Instituto Dante Pazzanese de Cardiologia

5. Título de Especialista em Medicina Intensiva pela AMIB-AMB.

6. Professora de Patologia Clínica da UNIFESP/EPM.

7. Professor Titular da Disciplina de Clínica Médica da UNIFESP/EPM.
}

*Recebido da Unidade de Terapia Intensiva da Disciplina de Clínica Médica da Universidade Federal de São Paulo da Escola Paulista de Medicina (UNIFESP-EPM), São Paulo, SP.

Apresentado em 24 de maio de 2007

Aceito para publicação em 19 de novembro de 2007

Endereço para correspondência:

Dra. Ticiana Paes

Rua Napoleão de Barros, 715, 3A - Vila Clementino

04024-002 São Paulo, SP

E-mail: ticipaes@hotmail.com

(C)Associação de Medicina Intensiva Brasileira, 2007
(Ur): 167, Na: 133, K: 6.9, glicose: 561; gasometria arterial (cateter de oxigênio: $2 \mathrm{~L} / \mathrm{min}$ ): $\mathrm{pH}: 6.93, \mathrm{pCO}_{2}: 12.1, \mathrm{pO}_{2}$ : 107, BE: $-28,8, \mathrm{HCO}_{3}: 2,4$, Sat $91,3 \%$, lact: 79 ; urina I: pH: 6,0; leucócitos: 13; densidade: 1015; eritrócitos: 19; proteína: ++; glicose: +++; bilirrubina: negativa; corpos cetônicos: + denotando cetonemia. Eletrocardiograma com onda $T$ apiculada, bloqueio de ramo direito. A paciente foi tratada com insulina, hidratação, bicarbonato de sódio e introduzido antibioticoterapia. Após o tratamento inicial, os exames laboratoriais mostraram: Cr: 2,2, Ur: 122, Na: 162, K: 4,3, Ca: 6,4, glicose: 504, $\mathrm{pH}: 7,01, \mathrm{HCO}_{3}$ : 7.1, BE: - 22. Um dia após, a paciente apresentou importante dor abdominal acompanhada de irritação peritoneal, além de sonolência e dificuldade para falar; exames laboratoriais mostraram: $\mathrm{pH}: 7,4$, $\mathrm{pCO}_{2}: 31, \mathrm{pO}_{2}: 68, \mathrm{BE}:-4,4, \mathrm{HCO}_{3}: 19$, Sat.O $:$ : 93,5\%; Ur: 95; Cr: 1,4, albumina: 2,4, Ca: 0,95, Na: 166, K:4, bilirrubina: 0,5, bilirrubina D/l: 0,2/0,3, amilase: 1157, Gama-GT: 56, AST 7.210, ALT: 2.470, VHS: 15, lipase: 84. Ultrasonografia abdominal não apresentou alterações significativas. Evoluiu com importante piora clínica, parada cardiorrespiratória e óbito. A necrópsia evidenciou múltiplas áreas de infarto hepático, trombose vascular, infarto esplênico, congestão visceral e aterosclerose de aorta e seus ramos e pâncreas normal.

CONCLUSÕES: Os mecanismos de infarto hepático e esplênico neste caso não foram bem elucidados. Alguns fatores devem ter contribuído, tais como: desidratação e hipotensão devido a episódios de vômitos e febre que contribuem para diminuição do fluxo sanguíneo da veia porta e artéria hepática; o nível elevado de catecolaminas que ocorre em estado de hiperglicemia e cetoacidose metabólica pode induzir à vasoconstrição; aterosclerose difusa que é comumente vista em pacientes diabéticos e hipertensos. Este caso enfatiza a necessidade de investigar infarto hepático em pacientes diabéticos com cetonemia e com aumento de enzimas hepáticas. Anticoagulantes devem ser prontamente instituídos se houver trombose vascular.

Unitermos: diabete melito, necrose hepática; trombose de artéria hepática, trombose de veia porta 


\section{SUMMARY}

BACKGROUND AND OBJECTIVES: Hepatic infarction is characterized by parenchyma ischemic necrosis involving at least two acinis. It is extremely uncommon due to the arterial and portal venous blood supply. We report a case of a patient not know to have diabetes who developed massive areas of ischemic infarcts of the liver after episode of acutely diabetes decompensated.

CASE REPORT: A 67 year-old hypertensive female who has been presenting, for the last 10 days, polydipsia, high urinary volume, visual and gait impairment, nausea and vomiting was admitted to the emergency room (ER). During the physical examination it was observed dehydration, skin discoloration, peripheral cyanosis, hypothermia, tachycardia, hypotension and mild diffuse abdominal pain. Admissional laboratory exams demonstrated total leukocytes: 16.800, Cr: 3.7, Ur: 167, Na: 133, K: 6.9 , glucose: 561; arterial gasometry $\left(\mathrm{O}_{2}\right.$ catheter: $\left.2 \mathrm{~L} / \mathrm{min}\right): \mathrm{pH}: 6.93, \mathrm{pCO}_{2}: 12.1, \mathrm{pO}_{2}: 107$, B.E.: -28.8, $\mathrm{HCO}_{3}$ : 2.4, Sat $91.3 \%$, lactato: 79 ; urinalysis: pH: 6; leukocytes: 13; density: 1015; erythrocytes: 19; protein: ++; glucose: +++; bilirubin: negative; ketonic bodies: + denote ketonemia. EKG: sharp T wave, right branch block. Patient was treated with intravenous insulin, hydration, sodium bicarbonate and ceftriaxone. After initial treatment, the laboratory exams showed Cr: 2.2, Ur: 122, Na: 162, K: 4.3, Ca: 6.4, glucose: 504, $\mathrm{pH}: 7.01, \mathrm{HCO}_{3}$ : 7.1, B.E.: -22. One day after admission the patient presented with important abdominal pain and peritoneal irritation, followed by difficulty for talking and somnolence; routine laboratory exams showed arterial gasometry: $\mathrm{pH}: 7.4, \mathrm{pCO}_{2}: 31, \mathrm{pO}_{2}: 68, \mathrm{BE}$ : $-4.4, \mathrm{HCO}_{3}$ : 19, SatO $:$ : 93.5\%; Ur: 95,Cr: 1.4, albumin: 2.4, Ca: 0.95, Na: 166, K:4, bilirubin: 0.5, bilirubin D/l: 0.2/0.3, Amylase: 1157, Gamma-GT: 56, AST 7.210, ALT: 2.470, SR (sedimentation rate): 15, Lipase: 84 . Abdominal ultrasound was unremarkable. Patient respiratory function and conscience level worsened, requiring intubation. Despite all resuscitation efforts, she died. Necropsy showed multiple ischemic infarcts of the liver with vascular thrombosis, splenic infarcts, generalized visceral congestion and atherosclerosis of aorta and its branches. Pancreas was normal.

CONCLUSIONS: The mechanisms of hepatic and splenic infarctions in this case were unclear. The following factors may have contributed to necrosis: vomiting and fever should be considered to induce dehydration and hypotension, which further decrea- sed portal and hepatic arterial inflows; elevated level of catecholamine in hyperglycemic states might induce vasoconstriction effects; widespread atherosclerosis is commonly seen in diabetic and hypertensive patients. This case underlies the importance of searching for hepatic necrosis or infarction in any diabetic patient with elevated liver enzymes. Anticoagulation therapy should be instituted promptly upon recognition of vascular thromboses.

Key Words: diabetes mellitus, hepatic arterial thrombosis, hepatic necrosis, portal vein thrombosis

\section{INTRODUÇÃO}

O infarto hepático é caracterizado pela necrose isquêmica do parênquima hepático de, pelo menos, dois ácinos, acompanhado de insuficiência circulatória. $\mathrm{O}$ infarto hepático é extremamente incomum ${ }^{1-3}$. A raridade deste evento pode ser explicado pelo fluxo sangüíneo garantido por dupla irrigação para o fígado, proveniente da artéria hepática e da veia porta. Esse sistema permite uma via compensar a outra. Sendo assim, redução no aporte sangüíneo por ambas, veia porta ou pela artéria hepática, pode decorrer por outros fatores que limitariam os mecanismos compensatórios ${ }^{1-3}$. O objetivo deste estudo foi relatar um caso de paciente não sabidamente diabética que desenvolveu extensas áreas de necrose isquêmica hepática.

\section{RELATO DO CASO}

Paciente do sexo feminino, 67 anos, foi admitida no hospital por complicações no seu estado geral havia 10 dias, poliúria, polidipsia, borramento visual, inapetência, náuseas e vômitos. Antecedentes pessoais incluiam hipertensão, acidente vascular encefálico isquêmico (AVEi) havia dois anos e tabagismo. A paciente não era sabidamente diabética, segundo relato de familiares.

A paciente deu entrada no pronto socorro em mau estado geral, desidratada 4+/4+, taquicárdica (FC:136 bpm), pressão arterial inaudível. Nos exames da admissão mostraram acidose metabólica grave $(\mathrm{pH}: 6,9 \mathrm{e}$ bicarbonato: 2,4), lactato: 79 e glicose: 561 . A função renal mostrou-se alterada, caracterizando insuficiência renal com características de pré-renal (creatinina: 3,7 e uréia: 167), K: 6,9 e Na: 133. O hemograma mostrou hemoglobina: 13,3, hematócrito: 40, leucócitos: 16800 com $31 \%$ de bastões e $55 \%$ de segmentados. O exame de urina mostrou proteinúria $(2+)$, glicosúria $(3+)$ e 
corpos cetônicos (1+). Foram administrados hidratação vigorosa, insulina, antibioticoterapia e bicarbonato de sódio. Aos poucos foi revertida a acidose metabólica, a pressão arterial normalizou, a glicemia reduziu seus níveis, o potássio foi corrigido e a função renal foi melhorando gradativamente.

Porém, no dia seguinte, a paciente evoluiu com importante dor abdominal, hipotensão e sonolência. Os exames laboratoriais mostraram aumento de transaminases (TGO: 7210, TGP: 2470), aumento de amilase (1157) e bilirrubinas normais (BT: 0,5 BD/BI 0,2/0,3). A ultra-sonografia abdominal realizada no leito mostrou moderada quantidade de conteúdo anecóico na cavidade peritoneal, sem outras alterações.

A paciente evoluiu para acidose metabólica grave $(\mathrm{pH}$ : 6,9 e bicarbonato: 11,3), diminuição do nível de consciência, instabilidade hemodinâmica e parada cardiorrespiratória. Foram realizadas manobras de reanimação e suportes básico e avançado de vida sem sucesso.

A necropsia mostrou aterosclerose de aorta e ramos, necrose hepática submaciça e múltiplos infartos esplênicos (Figuras 1 e 2).

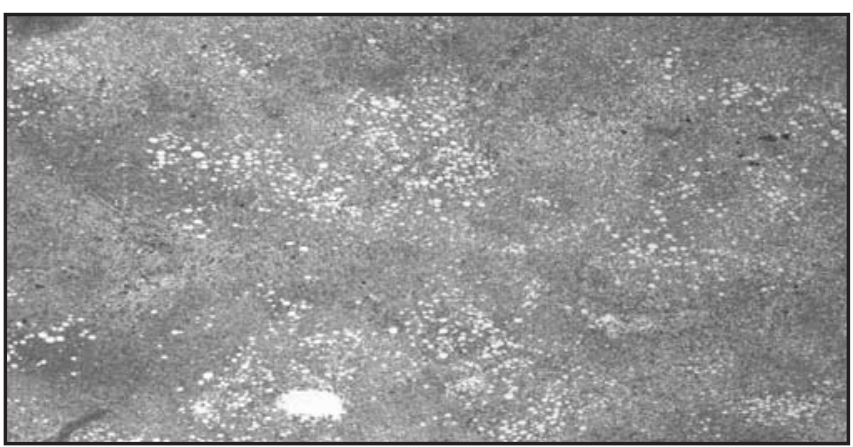

Figura 1 - Esteatose Microgoticular na Zona 3 e Necrose PanAcinar Acometendo Lóbulos Adjacentes.

Coloração (staining): HE-Aumento (Magnification): 40X

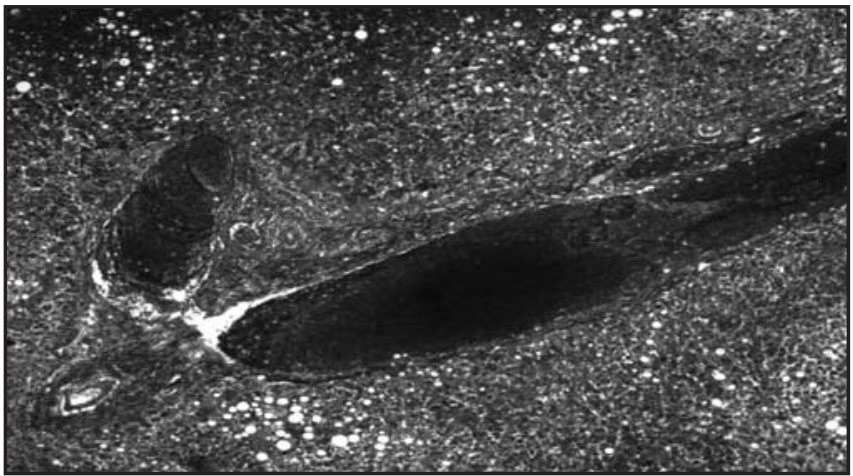

Figura 2 - Trombose em Vasos Portais com Necrose do Parênquima em Torno.

Coloração (staining): HE Aumento (Magnification): 200X

\section{DISCUSSÃO}

A necrose isquêmica hepática ocorre quando há o desequilíbrio entre a oferta e o consumo de oxigênio no tecido hepático ${ }^{4-6}$. Existem alguns mecanismos compensatórios como o aumento na taxa de extração de oxigênio pelos hepatócitos e o mecanismo de autoregulação do fluxo sanguíneo, que podem reduzir o impacto da hipoperfusão hepática. No entanto, para algumas situações de estresse, tais mecanismos são pouco eficazes, promovendo grave hipóxia hepatocelular, principalmente na zona 3, aparentemente mais vulnerável à baixa perfusão, por ser a mais distante do centro acinar (fluxo periportal) . $^{6}$

A artéria hepática é responsável por aproximadamente $35 \%$ do fluxo sangüíneo hepático e $50 \%$ do oxigênio necessário ao fígado, enquanto que a veia porta é responsável pelo restante ${ }^{6}$. Adicionalmente há uma extensa rede de artérias colaterais de menor calibre (possivelmente 26) que ajudam a suprir o fluxo sanguíneo distal do tecido hepático. Neste cenário, a oclusão da artéria hepática pode ser tolerada sem grandes conseqüências devido ao fluxo da veia porta, combinado a esta grande quantidade de colaterais existentes ${ }^{7}$. Esses fatores explicam o fato de um infarto hepático, causado por trombose de artéria hepática, ser evento mais raro que aquele causado pela trombose da veia porta.

Saegusa e col. ${ }^{3}$, em uma série de autópsias, relataram provável associação entre a ocorrência de relevantes disfunções hemodinâmicas e trombose venosa hepática, na maior parte dos casos de infarto hepático. Isso sugere que, em situações de disfunção do fluxo venoso portal, a hipotensão sistêmica pode levar à redução do fluxo arterial e à necrose parenquimatosa. Nessa série, a trombose arterial foi decorrente de procedimentos intervencionistas.

O infarto hepático foi descrito em vários outros relatos $^{4-6}$, relacionado a distintas causas como fatores iatrogênicos, procedimentos arteriais invasivos, cirurgia abdominal, escleroterapia endoscópica de varizes de esôfago, transplante hepático, embolização ou invasão tumoral, aneurisma de artéria hepática, ateromatose, sindrome hemolítica-urêmica, lupus eritematoso sistêmico, trauma abdominal fechado e diabete melito descompensada com presença de cetoacidose.

A descrição da associação entre necrose isquêmica e cetoacidose diabética foi feita por $\mathrm{Ng}$ e col. ${ }^{5}$ que, relataram um caso de paciente de 36 anos com infarto hepático documentado através de angiograma e bi- 
ópsia, após episódio de cetoacidose. Também Deng e col. ${ }^{6}$ descreveram um caso semelhante em paciente de 53 anos admitido no hospital, com diarréia e dor abdominal e diagnóstico comprovado, através de laparotmia, da presença de múltiplos infartos hepáticos; nesse mesmo relato, os autores consideraram o uso da glibenclamida como possível fator adicional de risco a lesão isquêmica.

O mecanismo exato de necrose hepática no caso relatado não foi totalmente elucidado. Alguns fatores, em conjunto, podem ter contribuído para esse evento. A desidratação e hipotensão podem diminuir a perfusão hepática e resultar em isquemia4: nesse caso, a desidratação e a hipotensão podem ser explicadas pelos episódios de vômitos e febre. A hipotensão arterial está freqüentemente implicada no desenvolvimento de infarto hepático ${ }^{1-3}$ já que reduz o fluxo tanto da veia porta quanto da artéria hepática.

Os níveis elevados de catecolaminas na cetacidose diabética podem levar a vasoconstrição promovendo isquemia e infarto: além disso, a diabete e a dislipidemia induzem disfunção endotelial e dificuldade para adaptação a situações de lesão ao estresse ${ }^{5}$.

$A$ aterosclerose pode também ser um fator contribuinte atuando em conjunto com a desidratação e com elevados níveis circulantes de catecolaminas favorecendo a isquemia hepática. Finalmente a instabilidade hemodinâmica, disfunção endotelial e estado de hipercoagulabilidade aumentam a probabilidade de trombose e diminui a fibrinólise, que pode ser encontrado em pacientes diabéticos com angiopatia ${ }^{6,7}$.

Esses fatores em conjunto poderiam explicar a necrose hepática submaciça e os múltiplos infartos esplênicos observados durante a necropsia da paciente.

A necrose isquêmica hepatica freqüentemente manifesta-se com dor abdominal, febre, elevação da contagem sérica de leucócitos e acentuado aumento de enzimas hepáticas; convém citar que a hiperglicemia dos pacientes diabéticos pode contribuir para a lesão do sistema nervoso autônomo visceral e conseqüente insensibilidade à dor isquêmica pode manifestar, a semelhança do infarto agudo do miocárdio, quadros de necrose hepática isquêmica sem dor .

A tomografia computadorizada e mais recentemente angiotomografia, além da angiografia podem contribuir fortemente para melhor delimitação do diagnóstico ${ }^{6-10}$. Nos casos onde é diagnosticada trombose de veia porta como a causa da necrose hepática, é importante introduzir tratamento anticoagulante imediatamente. A anticoagulação nesses casos reduz risco de eventos trombóticos em até dois terços. Isto afeta principalmente a recorrência de novos eventos e impede a extensão dessa trombose no território da veia porta e até de veia mesentérica ${ }^{8}$.

\section{CONCLUSÃO}

O infarto submaciço do fígado é um evento raro, devido ao rico suprimento sanguíneo hepático, além dos seus mecanismos compensatórios. No presente caso, houve inúmeros possíveis mecanismos, atuando em conjunto, para promoção da grave lesão hepatocelular e sua resultante insuficiência hepática fulminante, incompatível com a vida. Neste cenário, em pacientes diabéticos e com cetoacidose, o aumento de enzimas hepáticas e a dor abdominal devem ser avaliados com a possibilidade do diagnóstico de necrose isquêmica hepática ou infarto hepático e a terapia de anticoagulação deve ser considerada após a confirmação da trombose vascular.

\section{REFERÊNCIAS}

01. Woolling KR, Baggenstoss $\mathrm{AH}$, Weir JF - Infarcts of the liver. Gastroenterology, 1951;17:479-493.

02. Seeley TT, Blumenfeld CM, Ikeda R et al - Hepatic infarction. Human Pathol 1972;3:265-276.

03. Saegusa M, Takano Y, Okudaira M - Human hepatic infarction: histopathological and postmortem angiological studies. Liver, 1993;13:239-245.

04. Francque S, Condat B, Asselah T et al - Multifactorial aetiology of hepatic infarction: a case report with literature review. Eur J Gastroenterol Hepatol, 2004;16:411-415.

05. Ng RC, Sigmund CJ, Lagos JA et al - Hepatic infarction and diabetic ketoacidosis. Gastroenterology, 1977;73: 804-807.

06. Deng YG, Zhao ZS, Wang M et al - Diabetes mellitus with hepatic infarction: a case report with literature review. World J Gastroenterol, 2006;12:5091-5093.

07. Pathogenesis of liver injury in circulatory failure. Up to date, 2007. Versão 15.1 on line. Disponível em: http://www.utdol.com/application/search. asp. Acessado em 10 de abril de 2007.

08. Condat B, Pessione F, Hillaire S et al - Current outcome of portal vein thrombosis in adults : risk and benefit of anticoagulant therapy. Gastroenterology, 2001;120:490-497.

09. Wu Z, Lu Y, Zhang XG et al - Liver infarction: analysis of two cases. Gandan Waike Zazhi, 2002;10:288-290.

10. Sundaram M, Srivisal S, Lagos JA et al - Angiographic demonstration of non-occlusive hepatic infarction with scintigraphic and microscopic correlation. Gastrointest Radiol, 1978;15:3:39-42. 